indication. Liver bloods, clotting screen, bone profile tests were performed on $40 \%, 24 \%$ and $27 \%$ respectively in the second cycle.

The utility of blood tests may be an under recognised subject in paediatric training which needs addressing. This project typified positive impact of culture change via QI champions and educational sessions. Implementation of such changes is sustainable with an estimated savings of at least $£ 500 /$ month.

\section{CHILDREN'S EMERGENCY DEPARTMENT REVIEW CLINIC: WHAT WE HAVE LEARNT IN THE COVID-19 PANDEMIC}

Charlotte Munday, Neha Jain, Seamus Harrington, Benjamin Darvill, Sophie Perry, Alice Irwin, Francesca Deibel, Richard Turck, Clarissa Barber. Bristol Royal Hospital for Children

\subsection{6/emj-2020-rcemabstracts.54}

Aims/Objectives/Background The Children's Emergency Department (CED) in the Bristol Royal Hospital for Children (BRHC) runs 'Review Clinics' by a Paediatric Emergency Consultant. These were suspended in March 2020 due to the Covid-19 pandemic and new patient pathways were put in place. Our aim was to assess the effectiveness of these pathways and to evaluate if they should continue once the pressures of Covid-19 have eased.

Methods/Design We reviewed all the attendances to the ED Review Clinic over a six-week period in 2019 and identified common conditions that are seen in this clinic - abscesses, cellulitis, scaphoid fracture, toddler fracture, limp, headache, peri-orbital cellulitis, torticollis, and neck lumps (accounting for $66 \%$ of all attendances).

We then identified all of the ED attendances with these conditions over the same six-week period during the Covid-19 pandemic and performed a service review using medical notes. Results/Conclusions All children presenting with a limp during the Covid-19 pandemic were discharged with a leaflet and advised to return in one week if the limp persisted. None of them re-attended.

Scaphoid and toddler fractures that presented during the Covid-19 pandemic were followed-up by Orthopaedics with a combination of telephone and face-to-face reviews. The toddler fractures were put into soft casts to be removed at home - 93\% of parents were happy to do this. Orthopaedic review was quicker than the previous ED Review Clinic (an average of 63 hours compared to 216 for scaphoid fractures and 67 hours compared to 235 for toddler fractures).

The results suggest that ED Review Clinics can and should change. Children with a limp can be sent home safely with clear advice. There is a greater role for telephone reviews, in particular, for injuries such as toddler fractures. This would be quicker, less time-consuming for patients, parents and physicians and more cost effective for the department.

\section{PRESENTATION OF VULNERABLE CHILDREN TO ED DURING LOCKDOWN: A CONCERNING CONSEQUENCE OF ISOLATION}

Lalarukh Asim, Raeesa Jina, Sreena Das. King's College Hospital
Aims/Objectives/Background This study aims to evaluate changes to child safeguarding attendances to the emergency department (ED) during the lockdown period 1st of March till 31st May 2020 compared to the same time period in 2019.

The COVID-19 pandemic is the defining health crisis of our time and the first time firm social restrictions have been imposed since safeguarding practices have become embedded in the NHS. The NSPCC reported a large increase in contacts but there remain concerns that vulnerable children are invisible to agencies during this time.

Methods/Design All children ( $<18$ years) who attended King's College Hospital's ED, and were reviewed in the weekly ED safeguarding meeting were included. Data was collected from electronic patient records for different parameters.

Results/Conclusions The total number of children presenting via ED for safeguarding review fell from 865 in 2019 to 355 for the same period in 2020. However, the proportion requiring action by the hospital safeguarding team showed a significant increase $\left(p=1.5 \times 10^{-4}\right)$ suggesting the severity of cases during COVID-19 is worse.

The percentage of stabbings doubled $(\mathrm{p}=0.04)$ despite lockdown measures. This may be a contributor to the significant increase in referral to youth workers $\left(p=8 \times 10^{-4}\right)$. The number of children attending who were considered high risk due to previous safeguarding concerns dropped by $75 \%$.

As expected, the proportion of household injuries such as accidental ingestion and burns showed a significant increase $(p=0.01$ and $p=0.002$ respectively). The proportion of children from outside of our local boroughs was surprisingly higher in $2020(p=0.03)$.

The findings show that the number of cases triggering a safeguarding review has dropped during lockdown and raises concerns about vulnerable children who remain hidden. The findings also suggest an increased severity of safeguarding presentations, supporting our fears that the implications of lockdown on vulnerable children is yet to be realised.

\section{THE DIAGNOSTIC ROLE OF SHUNT SERIES RADIOGRAPHS (SSR) IN CHILDREN PRESENTING TO THE CHILDREN'S EMERGENCY DEPARTMENT (CED) WITH SUSPECTED VENTRICULOPERITONEAL (VP) SHUNT FAILURE}

${ }^{1}$ Angharad Griffiths, ${ }^{1}$ Ikechukwu Okafor, ${ }^{2}$ Thomas Beattie. ${ }^{1}$ Children's Health Ireland at Temple Street; ${ }^{2}$ University of Edinburgh

10.1136/emj-2020-rcemabstracts.56

Aims/Objectives/Background VP shunts are used to drain CSF from the cranial vault because of a wide range of pathologies and, like any piece of hardware, can fail. Traditionally investigations include SSR and CT. This project examines the role of SSR in evaluating children with suspected VP shunt failure.

Primary outcome: Sensitivity and specificity of SSR in children presenting to the CED with concern for shunt failure. Methods/Design Conducted in a single centre, tertiary CED of the national Irish Neurosurgical(NS) referral centre (ED attendance: $>50,000$ patients/year). 100 sequential SSR requested by the $\mathrm{CED}$ were reviewed. Clinical information was extracted from electronic requests. Shunt failure was defined by the need for NS intervention(Revision). 